\section{LESSON 11}

MARGINS:

Left, Pica 20; Elite 30;

Right, moved out of the way.

PAPER: Against the paper guide at 0 on the scale on the left. Turn up 7 single lines.

Check the 'ready-to-type' position: feet, body, fingers and eyes.

Warm up

Bounce your right thumb off the space bar.

Tap the keys sharply.

Keep your eyes on the copy.

x key

Practise the reach until you can type $x$ with the $s$ finger without looking down.

Keep either the a finger or the $f$ finger in position as you type $x$.

Keep your eyes on the copy.

Tap the keys sharply.

Comma (,) key

Practise the reach until you can type the comma with the $k$ finger without looking down. Keep the jl; fingers in position.

Tap the keys sharply but take care not to type the comma or the full stop too hard in case you make a hole in the paper.

\section{Consolidation}

Check the 'ready-to-type' position. Try to type each line in $1 / 2$ minute. Use the paper release lever when taking the paper out of the machine.

Centre and lock the carriage.

Cover the machine.

UNIT 3

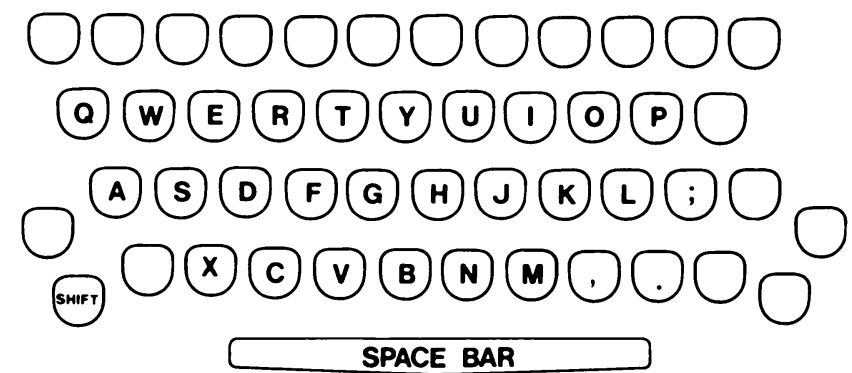

Remember to leave a blank line between sections.

Type the lines indicated by your teacher 3 times each.

1. Now it is up to you to say if you can do it or no.

2. Make haste; hurry it up. Last out must switch it.

3. Just as the clock was about to chime the man came.

4. Hugh has a quaint old van just like that one John.

5. I would like you to see my new cottages in oxford.

Type this line as many times as your teacher asks.

6. sss sxs sxs sxxf sxf fxs xsx sxs sxs asx asxf xsxs

Type the lines indicated by your teacher 3 times each.

7. ax wax ox box ox fox ex sex ax tax ix fixed ex vex

8. tax tax mix mix axed axed flex flex flax flax taxi

9. wash wax and tax the six taxis; fix the extra tax;

10. My extra taxi will fix the six extra jobs for you.

Gently type the comma. Type this line as many times as your teacher asks.

11. kkk k, k k, k, , k, k, k, k k, k it, is, if, not, $k,, k$

Type these lines 3 times each. Leave one space after a comma. Leave two spaces after a full stop at the end of a sentence.

12. that, that is, is, so, and, if, it, is, not, told, 13. Yes, do come. Please ask Ian. Now, before eight.

Leave one space following a full stop after a person's initials and the abbreviations for Mister (Mr.), Mistress (Mrs.) and Esquire (Esq.).

Type these lines 3 times each.

14. J. P. Lowe and N. H. James bought the best houses. 15. Mr. and Mrs. O. Y.. Hawkins came to the last party.

Type each line 3 times. Try to build up speed each time you repeat a line.

16. Once more, try again. Use mine, do not be afraid.

17. Peter fixed the quarter light. Mike made the tea.

18. Kate had six quaint dolls to put on her new stall.

19. Hugh said he thought the old car was out of sight.

20. Please, let Ian, Olive, Mark and Laura go on next.

LESSON 11 